\title{
Nursing Errors Regarding Preparation and Administration of Intravenous Drugs
}

\author{
Sabah Shaban Abd El salam ${ }^{1}$, Shalabia El Sayed Abo Zead ${ }^{2}$, Youssef Saleh ${ }^{3}$ Ghada Hassan Ahmed $^{4}$. \\ 1. B.Sc., Nursing Nursing Specialist, at Abnoub Secondary Nursing School, Assuit Governorate, Egypt. \\ 2. Professor of Medical Surgical Nursing, Faculty of Nursing, Assuit University Egypt. \\ 3. Professor of Plastic Surgery, Faculty of Medicine, Assuit University Egypt. \\ 4. Lecturer of Medical Surgical Nursing, Faculty of Nursing, Assiut University Egypt.
}

\begin{abstract}
Background: Intravenous (IV) medications pose particular risks because of their greater complexity and the multiple steps required in their preparation, administration and monitoring. The aim of this study was; assess the nursing errors during preparation and administration of intravenous drugs. Research design: Descriptive research design used in this study. Subjects: Non probability (convenience) sample was utilized, consisting of all nurses (30 nurses, 18 nurses in burn unit and 12 nurses in plastic surgery unit). Tools: Self-administered questionnaire sheet designed to assess nurses' knowledge in preparation and administration of intravenous drugs and direct Observational check list sheet designed to assess nurses' practice regarding the preparation and administration of IV drugs. Results: More than half of the studied nurses have good knowledge 53.3\% regarding preparation of IV drugs, $50 \%$ regarding Administration of IV drugs, more than half of them have adequate practice $60 \%$ and there were significant relation between nurses' demographic data and their nursing practice regarding training needs $(\mathrm{p}=0.042)$. Conclusion: more than half of the studied nurses have good knowledge and adequate practice. Recommendations The nurses need to continue attending training courses, and increase number of nurses in the units.
\end{abstract}

\section{Key words: Intravenous Drugs, Preparation \& Administration.}

\section{Introduction}

Medication errors in intravenous (IV) drug administration can be defined as any mistakes in the preparation, dispensing and administration of these drugs. Medication errors can be classified based on the stage of intravenous therapy in which the error occurs (pre-preparation phase, preparation of medication by nurse, drug labeling, and drug administration). In intravenously administered medications, errors may have particularly serious consequences (Strbova et al., 2015).

A narrative literature review estimates medication errors' percentage is included among $12 \%$ and $20 \%$ of total errors. Such errors are expensive from a human, economic, as well as social point of view (Frith, 2013).

The most frequent errors by category were wrong dose $(5.4 \%)$, wrong time $(3.7 \%)$, omission $(2.7 \%)$, unordered dose $(0.7 \%)$, and extra dose $(0.3 \%)$. Excluding wrong time errors, the error rate was $9.1 \%$ (Ding et al., 2015).

The lack of the pharmacological information, imprecise drug calculations, lack of observing the predetermined drug and pharmacological contracts, illegibility of physician prescriptions, the existence of drug names' similarities, drugs' improper packaging styles, drugs' packages and containers similarities are among the common medication errors, and reasons such as shortage of time, the lack or nonexistence of instruments and tools, and insufficient number of personnel indirectly affect medication errors (Pournamdar et al., 2016).

Studies that examined the types of medication errors divided them in categories, according to the description of the event: Omission error, wrong drug error, wrong patient error, wrong route error, wrong time error, wrong technique error, wrong dosage-form error and extra dose error. Thus, to avoid any type of medication error made by nurse, the implementation of preventive measures is undoubtedly beneficial. Nurses taking into account all precautions for medication errors, reduce firstly the incidence of medication errors, maintain the culture of safe hospital environment and ensure safe medications management by them (Fleming et al., 2016)

Intravenous administration of medications is one of the most common routes in burned patient particularly for those with a long length of hospitalization. Therefore, burned patients are at high risk for adverse drug events. Perhaps one of the most serious adverse events that can happen as a result of medication error is one that involves the IV route of administration as estimated that about one half of medication errors occurred in IV preparations and administrations, $\% 1$ of which resulted in serious adverse events (Abbasinazari et al., 2013).

\section{Aim of the Study}

This study aimed to assess the nurse's errors during preparation and administration of intravenous drugs. 


\section{Research question}

What are the common nurses errors during preparation and administration of intravenous drugs?

\section{Subjects \& Methods \\ Research design \\ Descriptive research design used in this study. \\ Technical design \\ Setting}

The study carried out in burn and plastic surgery unit at Assiut University Hospital.

Sample

Non probability (convenience) sample are utilized in this study, consisting of all nurses (30 nurse, 18 nurse in burn unit and 12 nurse in plastic surgery unit) working in burn and plastic surgery unit who are willing to participate in study, with age between 20 40 years and more than one year experience in burned unit.

Tools

Two tool used in this study and developed by the researcher to collect the necessary data for this study includes

Tool (I):Self-administered questionnaire sheet. It included two parts

Part (I): Demographic characteristics of nurses such as age, gender, years of work experience, marital status and previous training courses.

Part (II): Nurses knowledge in preparation and administration of intravenous drugs

This part was developed by the researcher based on literature review to assess nurse's knowledge in preparation and administration of intravenous drugs. It includes multiple of choice questions related to preparation and administration of intravenous drugs.

\section{Tool (II): Observational check list sheet}

It was developed by the researcher based on literature review to assess nurses' practice regarding the preparation and administration of IV drugs.

\section{Scoring System:}

1-Scoring system for nurse's knowledge in preparation and administration of intravenous drugs it consist of multiple of choice questions the true answer refers to 1 and the false answer refers to 0 and the score transformed into percent (\%) $<50 \%=$ poor, $50-70=$ fair, $>70=$ good.

2- Scoring system for nursing practice regarding preparation and administration of intravenous drugs Consist of: Done refers to 1 and not done refers to 0 and the score transformed into percent $(\%)<50 \%=$ poor, $50-70=$ fair $>70=$ good.

\section{Procedure}

It was concerned with construction and preparation of data collection tools. Managerial arrangement to carry out the study, where the investigator prepared formal requests to the directors of the study settings.
The purpose and the nature of the study were explained to gain their acceptance and support. Data collected by using a self-administered questionnaire sheet. All questionnaire items were explained by the investigator sufficiently. Interview with nurses was conducted to filling the self-administered questionnaire sheet for their knowledge, after filling the questionnaire the researcher was conduct direct observational check list sheet to assess nurses' practice regarding the preparation and administration of IV drugs.

Tools Validity and Reliability: The tools were tested for content validity by 5 expertise in the medical and medical surgical nursing field to ascertain relevance and competence \& reliability were tested by using Cronbach'scoaefficient alpha.

Pilot study:

Was done on $10 \%$ (3nurses) of the sample to assess the clarity, completeness and understanding of the tools. It also helped in the estimation of the time needed to fill the questionnaire, According to the results of the pilot study, tools was modified.

\section{Ethics Consideration:}

Research proposal was approved from ethical committee in the faculty of nursing.

1- There is no risk for study subject during application of the research.

2- The study was following the common ethical principles

3- Oral consent was obtained from mothers that were willing to participate in the study, after explaining the nature and purpose of the study.

4- Confidentiality and anonymity was assured.

5- Nurses had the right to refuse to participate and or withdraw from the study without any rational at any time.

6- Writing consent will be obtained from nurses that are willing to participate in the study, after explaining the nature of the study.

\section{Statistical analysis}

Data were analyzed by using the statistical package for social science (SPSS) version 23. Discrete data were expressed as frequency, percentage, Relation between variable was done using Chi- square test. Probability (P. value) less than 0.05 was considered significant and less than 0.001 was considered highly significant. 
Results

Table (1): Frequency distribution of demographic data for nurses participant $n=30$.

\begin{tabular}{|l|c|c|}
\hline \multicolumn{1}{|c|}{ Variable } & $\mathbf{N}$ & \% \\
\hline Age & & \\
\hline from20<30years & 12 & 40.0 \\
\hline from30 - 40years & 18 & 60.0 \\
\hline Gender & 2 & 6.7 \\
\hline Male & 28 & 93.3 \\
\hline Female & & \\
\hline Educational level & 27 & 90.0 \\
\hline Diploma & 3 & 10.0 \\
\hline Others & & \\
\hline Years of work experience & 6 & 20.0 \\
\hline from1< 3years & 4 & 13.3 \\
\hline from3 - 5years & 20 & 66.7 \\
\hline$>$ 5years & 20 & \\
\hline Previous training courses about the preparation and administration of IV fluids and medications \\
\hline Yes & 17 & 56.7 \\
\hline No & 13 & 43.3 \\
\hline
\end{tabular}

Table (2): Frequency distribution of nurses' knowledge about preparation and administration of intravenous drugs.

\begin{tabular}{|l|c|c|c|c|c|c|}
\hline \multicolumn{2}{|c|}{ Medication } & \multicolumn{2}{c|}{ Good } & \multicolumn{2}{c|}{ Fair } & \multicolumn{2}{c|}{ Poor } \\
\cline { 2 - 7 } & $\mathbf{N}$ & $\mathbf{\%}$ & $\mathbf{N}$ & $\mathbf{\%}$ & $\mathbf{N}$ & $\mathbf{\%}$ \\
\hline Nurses knowledge about IV medication & 16 & 53.3 & 10 & 33.3 & 4 & 13.3 \\
\hline Preparation of IV medication & 15 & 50.0 & 15 & 50.0 & 0 & 0.0 \\
\hline Administration of IV medication &
\end{tabular}

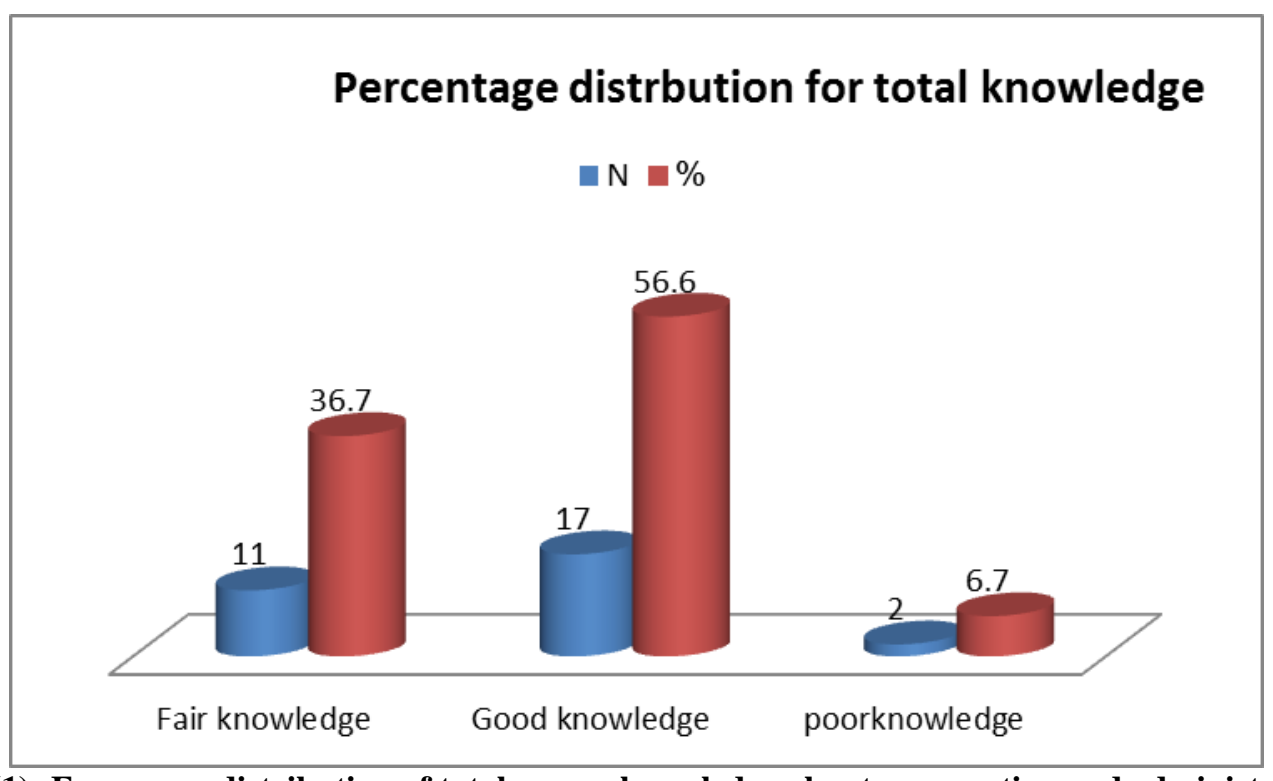

Figure (1): Frequency distribution of total nurses knowledge about preparation and administration of intravenous drugs. 
Table (3): Frequency distribution of total nurses practice about preparation and administration of intravenous drugs.

\begin{tabular}{|l|c|c|c|c|}
\hline \multicolumn{2}{|c|}{ Variables } & \multicolumn{2}{c|}{ Done } & \multicolumn{2}{c|}{ Not done } \\
\cline { 2 - 6 } & $\mathrm{N}$ & $\%$ & $\mathrm{~N}$ & \multicolumn{2}{c|}{} \\
\hline Nurses practice about medication & 21 & 70 & 15 & 50 \\
\hline Preparation and add medication to IV & 15 & 50.0 & 13 & 43.3 \\
\hline Preparation of medication from an ampule & 17 & 56.7 & 11 & 36.7 \\
\hline Preparation of medication from vial & 19 & 63.3 & 14 & 46.7 \\
\hline Administration of medication from an ampoule & 16 & 53.3 & & \\
\hline With drawing medication from a vial & & & & \\
\hline
\end{tabular}

Table (4): Total practice score for nurses $n=30$.

\begin{tabular}{|l|c|c|}
\hline \multicolumn{1}{|c|}{ Variables } & $\mathbf{N}$ & \% \\
\hline \hline Inadequate practice $<70 \%$ & 12 & 40.0 \\
\hline Adequate practice $>70 \%$ & 18 & 60.0 \\
\hline
\end{tabular}

Table (5): Relation between demographic data and nurses knowledge $\mathbf{n}=\mathbf{3 0}$.

\begin{tabular}{|c|c|c|c|c|c|c|c|}
\hline \multirow{3}{*}{ Variable } & \multicolumn{4}{|c|}{ Knowledge } & & & \multirow{3}{*}{ p.v } \\
\hline & \multicolumn{2}{|c|}{ Fair } & \multicolumn{2}{|c|}{ Good } & \multicolumn{2}{|c|}{ Poor } & \\
\hline & $\mathbf{N}$ & $\%$ & $\mathbf{N}$ & $\%$ & $\mathbf{N}$ & $\%$ & \\
\hline \multicolumn{8}{|l|}{ Age } \\
\hline from20tolessthan30years & 4 & 13.3 & 6 & 20.0 & 2 & 6.7 & \multirow[t]{2}{*}{$.200 \mathrm{~ns}$} \\
\hline from30to40years & 7 & 23.3 & 11 & 36.7 & 0 & 0.0 & \\
\hline \multicolumn{8}{|l|}{ Gender } \\
\hline Male & 1 & 3.3 & 1 & 3.3 & 0 & 0.0 & \multirow[t]{2}{*}{$.877 \mathrm{~ns}$} \\
\hline Female & 10 & 33.3 & 16 & 53.3 & 2 & 6.7 & \\
\hline \multicolumn{8}{|l|}{ Education Level } \\
\hline Diploma & 9 & 30.0 & 16 & 53.3 & 2 & 6.7 & \multirow[t]{2}{*}{$.506 \mathrm{~ns}$} \\
\hline Others & 2 & 6.7 & 1 & 3.3 & 0 & 0.0 & \\
\hline \multicolumn{8}{|l|}{ Years of work experience } \\
\hline from1 toless3years & 1 & 3.3 & 5 & 16.7 & 0 & 0.0 & \multirow[t]{3}{*}{$.315 \mathrm{~ns}$} \\
\hline from3to5years & 3 & 10.0 & 1 & 3.3 & 0 & 0.0 & \\
\hline morethan5years & 7 & 23.3 & 11 & 36.7 & 2 & 6.7 & \\
\hline \multicolumn{8}{|l|}{ Training about IV } \\
\hline Yes & 3 & 10.0 & 14 & 46.7 & 0 & 0.0 & \multirow[t]{2}{*}{$.004 * *$} \\
\hline No & 8 & 26.7 & 3 & 10.0 & 2 & 6.7 & \\
\hline
\end{tabular}

Chi-Square Tests $\quad * *=$ highly significance $* p \leq 0.01 \quad N s=$ Non significant difference P $>0.05$

Table 6: Relation between Nursing practice and demographic data $\quad \mathbf{n}=30$

\begin{tabular}{|c|c|c|c|c|c|}
\hline \multirow{3}{*}{ Variable } & \multicolumn{4}{|c|}{ Practice } & \multirow{3}{*}{ p.value } \\
\hline & \multicolumn{2}{|c|}{ inadequate } & \multicolumn{2}{|c|}{ Adequate } & \\
\hline & $\mathbf{N}$ & $\%$ & $\mathbf{N}$ & $\%$ & \\
\hline \multicolumn{6}{|l|}{ Age } \\
\hline from20tolessthan30years & 3 & 10.0 & 9 & 30.0 & \multirow{2}{*}{$.162 \mathrm{~ns}$} \\
\hline from30to40years & 9 & 30.0 & 9 & 30.0 & \\
\hline \multicolumn{6}{|l|}{ Gender } \\
\hline Male & 1 & 3.3 & 1 & 3.3 & \multirow{2}{*}{$.648 \mathrm{~ns}$} \\
\hline Female & 11 & 36.7 & 17 & 56.7 & \\
\hline \multicolumn{6}{|l|}{ Education Level } \\
\hline Diploma & 11 & 36.7 & 16 & 53.3 & \multirow{2}{*}{$.653 \mathrm{~ns}$} \\
\hline Others & 1 & 3.3 & 2 & 6.7 & \\
\hline
\end{tabular}




\begin{tabular}{|l|c|c|c|c|c|}
\hline \multirow{2}{*}{ Variable } & \multicolumn{4}{c|}{ Practice } & \multirow{2}{*}{ p.value } \\
\cline { 2 - 5 } & \multicolumn{2}{|c|}{ inadequate } & \multicolumn{2}{c|}{ Adequate } & \multirow{2}{*}{} \\
\hline & $\mathbf{N}$ & $\mathbf{\%}$ & $\mathbf{N}$ & $\mathbf{\%}$ & \\
\hline Years of work experience & & & & & \\
\hline from1toless3years & 1 & 3.3 & 5 & 16.7 & \multirow{2}{*}{$.420 \mathrm{~ns}$} \\
\hline from3to5years & 2 & 6.7 & 2 & 6.7 & \\
\hline morethan5years & 9 & 30.0 & 11 & 36.7 & \\
\hline Training about IV & & & & & \multirow{2}{*}{$.042 *$} \\
\hline Yes & 4 & 13.3 & 13 & 43.3 & \\
\hline No & 8 & 26.7 & 5 & 16.7 & \multirow{2}{*}{} \\
\hline
\end{tabular}

Chi-Square Tests

$*=$ Significant difference ${ }^{*} p \leq 0.05 \quad N s=$ Non significant difference $P>0.05$

Table (1): Shows that, female nurses were the majority of the studied sample; the highest percent of nurses' age was between 30-40yrs old. As regard educational level and years of work experience, the majority of them have a diploma degree $(90.0 \%)$. More than tow third of the studied nurses have experience more than 5 years during the work period (90\%, 66.7\% respectively). Regarding Previous training, more than half of them attending training courses about the preparation and administration of IV fluids and medications $(56.7 \%)$.

Table (2): This table show that, more than half of the studied nurses have good knowledge $53.3 \%$ regarding Preparation of IV medication, 50\% regarding Administration of IV medication.

Figure (1): Illustrated that, more than half of the studied nurses have good knowledge $>70 \%$ about preparation and administration of intravenous drugs.

Table (3): Shows that, the majority of the studied nurses done correctly steps related to preparation and adds medication to IV (70\%) and more than half of them done correctly steps related to administration of medication from an ampoule $(63.3 \%)$.

Table (4): Shows that more than half of the studied nurses have adequate practice $(60 \%)$.

Table (5): Shows that, there were highly significant difference between nurses demographic data and their knowledge regarding training about IV $(\mathrm{p}=0.04)$.

Table (6): Shows that, there were significant relation between nurses' demographic data and their nursing practice regarding training needs $(0.042)$.

\section{Discussion}

Medication administration errors occur frequently and are more likely to result in serious harm and death than other types of medication errors. Direct observational studies in hospitals have produced estimates of administration error rates of around 19$27 \%$ of drugs administered to patients. Errors can have negative impacts for both patients and nurses. A small proportion of errors will lead to serious patient outcomes and even minor errors can leave long- lasting effects on the nurses' involved (Westbrook et al., 2018).

So our study was designed to assess nurse's errors during preparation and administration of intravenous fluids and medications in burn and plastic surgery units.

The Discussion covered the main result finding as follows

I: Demographic characteristics for nurses

The present study results show that the majority of nurses were female; their age ranged from (30-40 years) As regard educational level and years of work experience, the majority of them have a diploma degree and more than tow third of them have experience more than 5 years during the work period; it was fond that more than half of them attending training courses about the preparation and administration of IV drugs.

This finding was supported by (Shamsuddin \& Shafie, 2012) who found in their study that most of gender profiles of respondents were females and their age ranged from 22-37 years. The majority of the respondents were diploma holders and less than half of respondents have less than 2 years nursing experience while less than one fifth of them have 10 years or more working experience. Also this finding is in disagreement, with the paper of (Fulbrook\&Albarran, 2012) which mentioned that, more than one third was female; about half was the age of equal or less than 25 years; majority of them held bachelor degree in nursing and were staff nurses; more than two thirds had nursing experience equals or less than 5 years.

II: Nurses knowledge during preparation and administration of intravenous drugs.

According to Nurses knowledge during preparation of intravenous drugs the present study clarified that, more than half of the studied nurses have good knowledge $53.3 \%$ regarding Preparation of medication, and $50 \%$ regarding Administration of medication, and there were highly significant difference between nurses demographic data and their knowledge regarding training about IV $(\mathrm{p}=0.04)$. 
The knowledge levels of nurses in this study were not influenced by age, years of work experience, and level of nursing education this may be due to having more than more than 5years of work experience, and more than half of studied nurses having Previous training courses about the preparation and administration of IV fluids and medications is similar to the study done by

(Shamsuddin \& Shafi, 2012).Also this study disagree with Sohrevardi et al., (2014) who suggested that: Lack of knowledge in use of drugs: Nurses are not always knowledgeable regarding the nature and volume of diluents, administration techniques, rates and schedules of administration, and physicochemical incompatibility. Wrong preparation by nurses: All medicines are prepared in the unit by the nurses. When the demand is intense, several nurses prepare the drugs for their own patients at the same time in cramped quarters. They are also frequently disturbed, which means they stop their preparations. The crowding and the interruptions increase the risk of error. Lack of standardization of protocols for preparation and administration: Since each nurse prepares and administers, medicines according to his/her knowledge and habits, there is heterogeneity of practice.

UN availability of medical devices: medical devices for intravenous administration are limited, and the lack of staff training and protocols in this area are also risk factors.

Lack of the presence of a pharmacist: Clinical pharmacists have been shown to improve the quality of patient care, for instance by reducing medication errors and (potential) adverse drug events.

Tromp \& Van (2009) illustrated that Observation methods for studying medication preparation and administration errors could have influenced the results. Even when all precautions are taken to minimize the effects of observation, it is still possible that the presence of a researcher may affect nurses' behavior. Some nurses told the observer that they miss some steps in the total process of administration when the observer is not there. Therefore, the error rate may have been even higher in the absence of the researcher. This implies that scores for the quality of the preparation and administration of intravenous drugs in this study are probably somewhat biased towards more positive scores.

(Shamsuddin \& Shafi, 2012) Suggested that many medication errors were found to be due to lack of pharmacological knowledge, improper attitude and skills in medication administration, $(5.41 \%)$ of the medication errors that occurred were due to failure in following the prescription and poor skills in administering drugs. The breakdown in communication and failure to follow policy are the main causes of medication errors.

III: Nurses practice during preparation and administration of intravenous drugs.

The finding of the present study was clarified that, the majority of the studied nurses done correctly steps related to preparation and adds medication to IV $(70 \%)$ and more than half of them done correctly steps related to administration of medication from an ampoule $(63.3 \%)$.and more than half of the studied nurses have adequate practice $(60 \%)$, and there were significant relation between nurses' demographic data and their nursing practice regarding training needs $(\mathrm{p}=0.042)$.

In a study done by Morgan et al., (2012) he revealed that each clinical practice area should also give new and experienced nurses protected time to improve their learning and professional development in drug calculation and administering medicines. This would produce more knowledgeable and confident practitioners create a safer environment for patients and increase patient and staff satisfaction.

\section{Conclusions}

Based on the results of the present study, it can be concluded that:

The majority of the studied nurses have good knowledge and done correctly steps related to preparation and add medication to IV (70\%) and more than half of them done correctly steps related to administration of medication from an ampoule $(63.3 \%)$.

\section{Recommendations}

The nurses need to continue attending training courses, increase number of nurses in the units to decrease the work load, and maintain adequate supply of hand washing material to follow aseptic techniques.

\section{References}

1. Abbasinazari, M., Talasaz, A. H., Mousavi, Z., \& Zare-Toranposhti, S. (2013). Evaluating the frequency of errors in preparation and administration of intravenous medications in orthopedic, general surgery and gastroenterology wards of a teaching hospital in Tehran. Iranian journal of pharmaceutical research: IJPR, 12(1), 229

2. Ahmad Fuad Shamsuddin \& Sarah Diyana Shafi e / Procedia(2012): -Knowledge of nurses in the preparation and administration ofintravenous medications Social and Behavioral Sciences $60602-609$

3. Ding, Q., Barker, K., Flynn, E., Westrick, S., Chang, M., Thomas, R., \& Sesek, R., (2015): 
Incidence of intravenous medication errors in a Chinese hospital. Value in Health Regional Issues, 6, 33-39.

4. Fleming, S., Brady, A., \& Malone, A., (2014): An evaluation of the drug calculation skills of registered nurses. Nurse Education in Practice, 14(1), 55-61.

5. Frith, K., (2013): Medication errors in the intensive care unit: literature review using the SEIPS model. AACN Adv Crit Care, 24(4), 389404..

6. Morgan A., (2012): Implementing structured preceptorship in an acute hospital. Nursing Standard; 26: 28, 35-39.

7. Pournamdar Z., Zare S., (2016): Survey of Medication Error Factors from Nurses' Perspective. Biol Med (Aligarh) 8: 310. doi: 10.4172/0974-8369.1000310

8. Sohrevardi S., Mirjalili M., Jarrahzadeh M., Mirjalili M., Mirzaei E., (2012): Evaluating the Frequency of Errors in Preparation and Administration of Intravenous Medications in the Intensive Care Unit of Shahid-Sadoughi Hospital in Yazd. J Pharm Care 2014; 2 (3): 114-119.

9. Strbova, P., Mackova, S., Miksova, Z., \& Urbanek, K., (2015): Medication errors in intravenous drug preparation and administration: a brief review. J Nurs Care, 4(285), 2167-1168.

10. Tromp, M., Natsch, S., \& Van Achterberg, T., Pharm World Sci (2009): 31: 413. https://doi.org/10.1007/s11096-008-9269-5

11. Westbrook, J., Rob, M., Woods, A., \& Parry, D., (2018): Errors in the administration of intravenous medications in hospital and the role of correct procedures and nurse experience. BMJ Qual Saf, 20(12), 1027-1034. Downloaded from http://qualitysafety.bmj.com/ on February 15, 2018 - Published by group.bmj.com. 